\title{
Arithmetics of beta-expansions
}

\author{
by \\ L. S. Guimond (Montréal), Z. Masáková (Praha) \\ and E. Pelantová (Praha)
}

1. Beta-expansions. Let $\beta$ be a real number strictly greater than 1 . A real number $x \geq 0$ can be represented using a sequence $\left(x_{i}\right)_{k \geq i>-\infty}$, $x_{i} \in \mathbb{Z}, 0 \leq x_{i}<\beta$, such that

$$
x=x_{k} \beta^{k}+x_{k-1} \beta^{k-1}+\ldots+x_{1} \beta+x_{0}+x_{-1} \beta^{-1}+x_{-2} \beta^{-2}+\ldots
$$

for some $k \in \mathbb{Z}$. We then write

$$
(x)_{\beta}=x_{k} x_{k-1} \ldots x_{1} x_{0} \bullet x_{-1} x_{-2} \ldots
$$

A particular representation is the $\beta$-expansion of $x$ (see [7]). The digits $x_{i}$ of the $\beta$-expansion are computed by the "greedy" algorithm: Let $[y]$ denote the largest integer smaller than or equal to $y$. Find $k \in \mathbb{Z}$ for which $\beta^{k} \leq$ $x<\beta^{k+1}$. Put $x_{k}=\left[x / \beta^{k}\right]$ and $r_{k}=x / \beta^{k} \bmod 1$. For $i \in \mathbb{Z}, i<k$ put $x_{i}=\left[\beta r_{i+1}\right]$ and $r_{i}=\beta r_{i+1} \bmod 1$. If $k<0$, i.e. $0<x<1$, we put $x_{0}, x_{1}, \ldots, x_{k+1}=0$ and write $(x)_{\beta}=0 \bullet 00 \ldots 0 x_{k} x_{k-1} \ldots$ If an expansion ends in infinitely many zeros, it is said to be finite and the final zeros are omitted.

We denote by $\operatorname{Fin}(\beta)$ the set of all $x$ for which $|x|$ has a finite $\beta$-expansion. The $\beta$-expansion of every $x \in \operatorname{Fin}(\beta)$ has therefore the form

$$
(x)_{\beta}=x_{k} x_{k-1} \ldots x_{1} x_{0} \bullet x_{-1} x_{-2} \ldots x_{-l},
$$

where $x_{k} x_{k-1} \ldots x_{1} x_{0} \bullet$ is the $\beta$-integer part and $\bullet x_{-1} x_{-2} \ldots x_{-l}$ is the $\beta$ fractional part of $x$. We usually call it simply the integer and the fractional part of $x$. The length of the fractional part of $x$ is denoted by $\mathrm{fp}_{\beta}(x)$. Elements of $\operatorname{Fin}(\beta)$ with vanishing fractional part (i.e. $\mathrm{fp}_{\beta}(x)=0$ ) are called $\beta$-integers. The set of $\beta$-integers is denoted by $\mathbb{Z}_{\beta}$.

The sets $\mathbb{Z}_{\beta}$ and $\operatorname{Fin}(\beta)$ are generally not closed under addition and multiplication. In spite of that it is sometimes useful in computer science to consider these operations in $\beta$-arithmetics. That is why it is important to

2000 Mathematics Subject Classification: 11R04, 11R06. 
study what fractional parts may appear as a result of addition and multiplication of $\beta$-integers.

Definition 1.1. Let $\beta>1$. We define

$$
\begin{aligned}
& L_{\oplus}(\beta):=\min \left\{L \in \mathbb{N}_{0} \mid \forall x, y \in \mathbb{Z}_{\beta}, x+y \in \operatorname{Fin}(\beta) \Rightarrow \operatorname{fp}_{\beta}(x+y) \leq L\right\}, \\
& L_{\odot}(\beta):=\min \left\{L \in \mathbb{N}_{0} \mid \forall x, y \in \mathbb{Z}_{\beta}, x y \in \operatorname{Fin}(\beta) \Rightarrow \operatorname{fp}_{\beta}(x y) \leq L\right\} .
\end{aligned}
$$

The minimum of an empty set is defined to be $+\infty$.

The aim of this paper is to give some quantitative results for $L_{\oplus}(\beta)$ and $L_{\odot}(\beta)$. Let us mention some of the known results. Frougny and Solomyak in [4] showed that $L_{\oplus}(\beta)$ is finite if $\beta$ is a Pisot number. A Pisot number $\beta$ is an algebraic integer such that $\beta>1$ and all its algebraic conjugates are of modulus smaller than 1 . Let us mention that to our knowledge no example is known of a $\beta$ such that $L_{\oplus}(\beta)$ or $L_{\odot}(\beta)$ is infinite.

Results for the special case of quadratic Pisot units are found in [3]. The authors gave exact values for $L_{\oplus}(\beta)$ and $L_{\odot}(\beta)$ when $\beta>1$ is a solution either of the equation $x^{2}=m x-1, m \in \mathbb{N}, m \geq 3$ or of the equation $x^{2}=m x+1, m \in \mathbb{N}$. In the first case $L_{\oplus}(\beta)=L_{\odot}(\beta)=1$; in the second case $L_{\oplus}(\beta)=L_{\odot}(\beta)=2$.

In this article we provide estimates on $L_{\oplus}(\beta)$ and $L_{\odot}(\beta)$ for those algebraic numbers $\beta>1$ that have at least one conjugate of modulus smaller than 1. Other results are valid for Pisot numbers $\beta$. The last part of the paper is devoted to quadratic Pisot numbers. We recover the results of [3] as a special case.

2. Beta-integers and cut-and-project sequences. The Rényi development of unity plays an important role in the description of the properties of the sets $\mathbb{Z}_{\beta}$ and $\operatorname{Fin}(\beta)$. For its definition we introduce the transformation $T_{\beta}(x):=\{\beta x\}$ for $x \in[0,1]$. The Rényi development of unity is defined as

$$
d(1, \beta):=t_{1} t_{2} \ldots t_{i} \ldots, \quad \text { where } t_{i}:=\left[\beta T_{\beta}^{i-1}(1)\right] .
$$

Parry [6] showed that $x=x_{k} x_{k-1} \ldots x_{1} x_{0} \bullet x_{-1} \ldots x_{-p}$ is a $\beta$-expansion if and only if $x_{i} x_{i-1} \ldots x_{-p}$ is lexicographically smaller than $t_{1} t_{2} \ldots t_{i} \ldots$ for every $-p \leq i \leq k$.

$\operatorname{Fin}(\beta)$ and $\mathbb{Z}_{\beta}$ are centrally symmetric sets. While $\operatorname{Fin}(\beta)$ is dense in $\mathbb{R}, \mathbb{Z}_{\beta}$ has no accumulation points. Distances between consecutive points in $\mathbb{Z}_{\beta}$ take values in $\left\{0 \bullet t_{i} t_{i+1} \ldots \mid i \in \mathbb{N}\right\}$. It is obvious that if $d(1, \beta)$ is eventually periodic, then $\mathbb{Z}_{\beta}$ has a finite number of distances between consecutive points. Numbers $\beta$ with this property are called beta-numbers. Some results and conjectures on beta-numbers are given in $[2,8]$; a description of beta-numbers is provided in [9]. Note that every Pisot number $\beta$ is a beta-number. 
The set $\mathbb{Z}_{\beta}$ of $\beta$-integers forms a ring only if $\beta$ is a rational integer, $\beta>1$. If $\beta$ is an algebraic integer of order $q \geq 2$, then $\mathbb{Z}_{\beta}$ can be naturally embedded into the ring $\mathbb{Z}[\beta]$ defined as

$$
\mathbb{Z}[\beta]:=\left\{n_{0}+n_{1} \beta+\ldots+n_{q-1} \beta^{q-1} \mid n_{i} \in \mathbb{Z}\right\} .
$$

Note that the ring $\mathbb{Z}[\beta]$ is dense in $\mathbb{R}$. In certain cases $\mathbb{Z}[\beta]$ coincides with $\operatorname{Fin}(\beta)$, i.e. $\operatorname{Fin}(\beta)$ is a ring (see [4]). Let us show that for $\beta$ an algebraic integer, the ring $\mathbb{Z}[\beta]$ is a projection of an integer lattice $\mathbb{Z}^{q} \subset \mathbb{R}^{q}$ on a one-dimensional subspace $V_{1}$ for a suitable decomposition $V_{1} \oplus V_{2}$ of the space $\mathbb{R}^{q}$. A similar construction can be found in [1].

Denote by $\beta^{(1)}=\beta, \beta^{(2)}, \ldots, \beta^{(s)}$ the real roots of the minimal polynomial of $\beta$ and by $\beta^{(s+1)}, \beta^{(s+2)}, \ldots, \beta^{(q-1)}, \beta^{(q)}$ the non-real conjugates of $\beta$. We have ordered the complex roots in such a way that $\overline{\beta^{(s+1)}}=\beta^{(s+2)}$, $\ldots, \overline{\beta^{(q-1)}}=\beta^{(q)}$.

First we have to find (possibly) complex vectors

$$
\left(\vec{x}^{(1)}\right)^{T}=\left(x_{0}^{(1)}, x_{1}^{(1)}, \ldots, x_{q-1}^{(1)}\right), \quad \ldots, \quad\left(\vec{x}^{(q)}\right)^{T}=\left(x_{0}^{(q)}, x_{1}^{(q)}, \ldots, x_{q-1}^{(q)}\right),
$$

such that for any $\vec{x}=\left(n_{0}, n_{1}, \ldots, n_{q-1}\right) \in \mathbb{R}^{q}$ we have

$$
\begin{aligned}
\vec{x}= & \left(\sum_{i=0}^{q-1} n_{i}\left(\beta^{(1)}\right)^{i}\right) \vec{x}^{(1)}+\left(\sum_{i=0}^{q-1} n_{i}\left(\beta^{(2)}\right)^{i}\right) \vec{x}^{(2)}+\ldots \\
& +\left(\sum_{i=0}^{q-1} n_{i}\left(\beta^{(q)}\right)^{i}\right) \vec{x}^{(q)} .
\end{aligned}
$$

Denote by $\mathbb{X}$ the $q \times q$ matrix with $(\mathbb{X})_{i j}=x_{j}^{(i)}$. Then (1) holds for each $\vec{x}$ if and only if

$$
I_{q}=\mathbb{V}\left(\beta^{(1)}, \ldots, \beta^{(q)}\right) \cdot \mathbb{X}
$$

where $\mathbb{V}\left(\beta^{(1)}, \ldots, \beta^{(q)}\right)$ is the Vandermonde matrix in variables $\beta^{(1)}, \ldots, \beta^{(q)}$,

$$
\mathbb{V}\left(\beta^{(1)}, \ldots, \beta^{(q)}\right):=\left(\begin{array}{cccc}
1 & 1 & \ldots & 1 \\
\beta^{(1)} & \beta^{(2)} & \ldots & \beta^{(q)} \\
\left(\beta^{(1)}\right)^{2} & \left(\beta^{(2)}\right)^{2} & \ldots & \left(\beta^{(q)}\right)^{2} \\
\vdots & \vdots & \vdots & \vdots \\
\left(\beta^{(1)}\right)^{q-1} & \left(\beta^{(2)}\right)^{q-1} & \ldots & \left(\beta^{(q)}\right)^{q-1}
\end{array}\right) .
$$

The determinant of $\mathbb{V}\left(\beta^{(1)}, \ldots, \beta^{(q)}\right)$ is equal to $\prod_{q \geq i>j \geq 1}\left(\beta^{(i)}-\beta^{(j)}\right)$. Since all conjugates are distinct, the determinant is non-zero.

Using the Cramer rule to compute $x_{j}^{(i)}$, we find that $\vec{x}^{(i)}$ is real if $\beta^{(i)}$ is real, and if $\beta^{(j)}$ and $\beta^{(j+1)}$ are complex conjugate roots then $\vec{x}^{(j)}=\overline{\vec{x}^{(j+1)}}$. 
Thus we can define a real basis $\vec{y}^{(1)}, \ldots, \vec{y}^{(q)}$ of $\mathbb{R}^{q}$ in such a way that $\vec{y}^{(i)}=\vec{x}^{(i)}$ if $\vec{x}^{(i)}$ is a real vector, and $\vec{y}^{(j)}=\vec{x}^{(j)}+\overline{\vec{x}^{(j)}}, \vec{y}^{(j+1)}=i\left(\vec{x}^{(j)}-\overline{\vec{x}}^{(j)}\right)$, if $\vec{x}^{(j)}$ and $\vec{x}^{(j+1)}=\overline{\vec{x}}^{(j)}$ are complex conjugate vectors.

Note that the coordinates of a vector $\vec{x}=\left(n_{0}, n_{1}, \ldots, n_{q-1}\right) \in \mathbb{R}^{q}$ with respect to the basis $\vec{y}^{(1)}, \ldots, \vec{y}^{(q)}$ are

$$
\begin{array}{ll}
\sum_{p=0}^{q-1} n_{p}\left(\beta^{(i)}\right)^{p} & \text { if } \vec{y}^{(i)}=\vec{x}^{(i)}, \\
\Re\left[\sum_{p=0}^{q-1} n_{p}\left(\beta^{(j)}\right)^{p}\right] & \text { if } \vec{y}^{(j)}=\vec{x}^{(j)}+\overline{\vec{x}^{(j)}}, \\
\Im\left[\sum_{p=0}^{q-1} n_{p}\left(\beta^{(j)}\right)^{p}\right] & \text { if } \vec{y}^{(j)}=i\left(\vec{x}^{(j)}-\overline{\vec{x}^{(j)}}\right) .
\end{array}
$$

If we put $V_{1}=\mathbb{R} \vec{y}^{(1)}$ and $V_{2}=\mathbb{R} \vec{y}^{(2)}+\mathbb{R} \vec{y}^{(3)}+\ldots+\mathbb{R} \vec{y}^{(q)}$, then the set $\mathbb{Z}[\beta]$ is the projection of $\mathbb{Z}^{q}$ on $V_{1}$ along $V_{2}$.

Projections of crystallographic lattices and non-crystallographic lattices are studied by the theory of cut-and-project sets. Let us recall a special case of their definition, which will be used here.

DeFinition 2.1. Let $U_{1}$ and $U_{2}$ be linear subspaces of $\mathbb{R}^{d}$ such that $\operatorname{dim} U_{1}=1, \operatorname{dim} U_{2}=d-1$ and $U_{1} \oplus U_{2}=\mathbb{R}^{d}$. Denote by $\pi_{1}$ the projection on $U_{1}$ along $U_{2}$ and by $\pi_{2}$ the projection on $U_{2}$ along $U_{1}$. Let $\Omega \subset U_{2}$ be a bounded set with non-empty interior $\Omega^{\circ}$, such that the closures of $\Omega$ and $\Omega^{\circ}$ coincide. If the mapping $\pi_{1}: \mathbb{Z}^{q} \rightarrow \pi_{1}\left(\mathbb{Z}^{d}\right)$ is one-to-one and $\pi_{2}\left(\mathbb{Z}^{d}\right)$ is dense in $V_{2}$, then the set $\Sigma(\Omega)=\left\{\pi_{1}(x) \mid x \in \mathbb{Z}^{d}, \pi_{2}(x) \in \Omega\right\}$ is called a cut-and-project set with acceptance window $\Omega$.

Basic properties of cut-and-project sets can be found in [5]. For us the most important property is that $\Sigma(\Omega)$ is relatively dense and uniformly discrete, i.e. there exists a real increasing sequence $\left(\alpha_{n}\right)_{n \in \mathbb{Z}}$ and constants $r, R>0$ such that $\Sigma(\Omega)=\left\{\alpha_{n} \vec{y} \mid n \in \mathbb{Z}\right\}$ and $r \leq \alpha_{n+1}-\alpha_{n} \leq R$ for all $n \in \mathbb{Z}$. In particular, the distances between consecutive points of $\Sigma(\Omega)$ take only finitely many values, i.e. the set $\left\{\alpha_{n+1}-\alpha_{n} \mid n \in \mathbb{Z}\right\}$ is finite.

Let us consider again an algebraic integer $\beta$ of order $q$ and the decomposition $\mathbb{R}^{q}=V_{1} \oplus V_{2}$ as described above. As shown by Akiyama [1], the projection $\pi_{1}\left(\mathbb{Z}^{2}\right)=\mathbb{Z}[\beta]$ of $\mathbb{Z}^{2}$ on $V_{1}$ is one-to-one and the projection $\pi_{1}\left(\mathbb{Z}^{2}\right)$ on $V_{2}$ is dense in $V_{2}$. For $\alpha \in \mathbb{Q}[\beta]$ we denote by $\alpha^{(k)}$ the image of $\alpha$ under the $k$ th Galois isomorphism $\mathbb{Q}[\beta] \rightarrow \mathbb{Q}\left[\beta^{(k)}\right]$ induced by the assignment $\beta \mapsto \beta^{(k)}$, i.e. if $\alpha=\sum_{i=0}^{q-1} n_{i} \beta^{i}$ for $n_{i} \in \mathbb{Q}$, then $\alpha^{(k)}=\sum_{i=0}^{q-1} n_{i}\left(\beta^{(k)}\right)^{i}$.

We shall focus on specific acceptance windows $\Omega(h) \subset V_{2}$ for $h>0$. As the acceptance window $\Omega(h) \subset V_{2}$ we choose the cartesian product of onedimensional line-segments $\left\{t \vec{y}^{(i)}|| t \mid<h\right\}$ if $\beta^{(i)}$ is real and two-dimensional 
ellipses $\left\{t \vec{y}^{(j)}+s \vec{y}^{(j+1)} \mid t^{2}+s^{2}<h^{2}\right\}$ if $\beta^{(j)}$ and $\beta^{(j+1)}$ are complex conjugate. Such an acceptance window $\Omega(h)$ satisfies the assumptions of Definition 2.1.

The point $\alpha \vec{y}^{(1)}$ belongs to $\Sigma(\Omega(h))$ if and only if $\alpha \in \mathbb{Z}[\beta]$ and $\left|\alpha^{(k)}\right|<h$ for $k=2, \ldots, q$. In other words, we have the following proposition.

Proposition 2.2. Let $\beta$ be an algebraic integer of order $q$. If $h>0$, then the set

$$
\Sigma(h)=\left\{\alpha \in \mathbb{Z}[\beta]|| \alpha^{(k)} \mid<h, k=2, \ldots, q\right\}
$$

is relatively dense and uniformly discrete and the distances in $\Sigma(h)$ take only finitely many values.

In the following, the sets $\Sigma(h)$ are called cut-and-project sequences. In the case that $\beta$ is a Pisot number, we show the relation between cut-and-project sequences and $\beta$-integers $\mathbb{Z}_{\beta}$.

Proposition 2.3. Let $\beta$ be a Pisot number of order $q$. Set

$$
l=[\beta] \max \left\{\left(1-\left|\beta^{(i)}\right|\right)^{-1} \mid i=2, \ldots, q\right\} .
$$

Then

$$
\mathbb{Z}_{\beta} \subset \Sigma(l), \quad \mathbb{Z}_{\beta}+\mathbb{Z}_{\beta} \subset \Sigma(2 l), \quad \mathbb{Z}_{\beta} \mathbb{Z}_{\beta} \subset \Sigma\left(l^{2}\right) .
$$

Proof. Let $x \in \mathbb{Z}_{\beta}$, i.e. $x= \pm \sum_{i=0}^{n} x_{i} \beta^{i}$ for some $n$. Then

$$
\left|x^{(j)}\right| \leq \sum_{i=0}^{n}[\beta]\left|\beta^{(j)}\right|^{i}<[\beta] \frac{1}{1-\left|\beta^{(j)}\right|} \leq l \quad \text { for } j=2, \ldots, q .
$$

The statement follows easily.

3. Sufficient conditions for finiteness of $L_{\oplus}$ and $L_{\odot}$. In this section we provide sufficient conditions on $\beta$ so that $L_{\oplus}(\beta)$ and $L_{\odot}(\beta)$ are finite. First we demonstrate Theorem 3.1 stating that $L_{\oplus}(\beta)$ and $L_{\odot}(\beta)$ are finite for a Pisot $\beta$. The statement for $L_{\oplus}$ has been proven in [4], but we provide a different and simpler proof. We further show that this condition is not necessary. Theorem 3.3 provides a different sufficient condition together with bounds on $L_{\oplus}(\beta)$ and $L_{\odot}(\beta)$. In the next section we apply Theorem 3.3 to the case of quadratic Pisot numbers.

Theorem 3.1. Let $\beta$ be a Pisot number. Then $L_{\oplus}(\beta)$ and $L_{\odot}(\beta)$ are finite.

Proof. Let $x, y \in \mathbb{Z}_{\beta}$. To determine $L_{\odot}(\beta)$ it suffices to consider $x, y>0$. Set $z_{0}=\max \left\{z \in \mathbb{Z}_{\beta} \mid z \leq x y\right\}$ and $r:=x y-z_{0}$. Since distances in $\mathbb{Z}_{\beta}$ are bounded by 1 , we have $0 \leq r<1$. Therefore obviously the remainder $r$ is the fractional part of the $\beta$-expansion of $x y$, i.e. $x y \in \operatorname{Fin}(\beta)$ if and only if $r \in \operatorname{Fin}(\beta)$. Since $l>1$, we have $\Sigma(l) \subset \Sigma\left(l^{2}\right)$ and according to Proposition 2.3 both $x y$ and $z_{0}$ belong to $\Sigma\left(l^{2}\right)$. 
According to Proposition 2.2 distances in $\Sigma\left(l^{2}\right)$ take only finitely many values, say $f_{1}, \ldots, f_{T}$. The gap $r$ between $z_{0}$ and $x y$ must be composed of these distances. Therefore $1>r=x y-z_{0}=\sum h_{i} f_{i}$, where $h_{i} \in \mathbb{N}_{0}$. Fractional parts of all results of multiplication $x y$ belong to the set

$$
F:=\left\{\sum_{i} h_{i} f_{i}<1 \mid h_{i} \in \mathbb{N}_{0}\right\}
$$

which is finite and therefore

$$
L_{\odot}(\beta) \leq \max \left\{\operatorname{fp}_{\beta}(r) \mid r \in F \cap \operatorname{Fin}(\beta)\right\} .
$$

To derive the finiteness of $L_{\oplus}(\beta)$ one uses an analogous argument.

A simple consequence of the above proof is that $\mathbb{Z}_{\beta}$ is a Meyer set.

Corollary 3.2. Let $\beta$ be a Pisot number. Then there exists a finite set F such that

$$
\mathbb{Z}_{\beta}+\mathbb{Z}_{\beta} \subset \mathbb{Z}_{\beta}+F, \quad \mathbb{Z}_{\beta} \mathbb{Z}_{\beta} \subset \mathbb{Z}_{\beta}+F .
$$

Theorem 3.1 gives a sufficient condition for finiteness of $L_{\oplus}(\beta)$ and $L_{\odot}(\beta)$. An upper bound on $L_{\oplus}(\beta)$ is determined in [10] using some complicated techniques. However, their result applies only to a class of Pisot numbers. The condition that $\beta$ is Pisot is however not necessary. In the following theorem we provide a similar estimate on $L_{\oplus}(\beta)$ with less restrictive criteria for $\beta$. Moreover, we determine an upper bound for $L_{\odot}(\beta)$.

THEOREM 3.3. Let $\beta>1$ be an irrational algebraic number such that at least one of its conjugates, say $\beta^{\prime}$, is of modulus smaller than 1 . Define

$$
H=\sup \left\{\left|z^{\prime}\right| \mid z \in \mathbb{Z}_{\beta}\right\}, \quad K=\inf \left\{\left|z^{\prime}\right| \mid z \in \mathbb{Z}_{\beta}, z \notin \beta \mathbb{Z}_{\beta}\right\} .
$$

If $K>0$, then $L_{\oplus}(\beta)$ and $L_{\odot}(\beta)$ are finite and

$$
\begin{aligned}
& \left(\frac{1}{\left|\beta^{\prime}\right|}\right)^{L_{\oplus}(\beta)}<\frac{2 H}{K}, \\
& \left(\frac{1}{\left|\beta^{\prime}\right|}\right)^{L_{\odot}(\beta)}<\frac{H^{2}}{K} .
\end{aligned}
$$

Proof. Let $x, y \in \mathbb{Z}_{\beta}$ and $x+y \in \operatorname{Fin}(\beta), x+y=\sum_{i=-L}^{k} a_{i} \beta^{i}, a_{-L} \geq 1$. Then $\beta^{L}(x+y) \in \mathbb{Z}_{\beta}$ and $\beta^{L}(x+y) \notin \beta \mathbb{Z}_{\beta}$. Thus

$$
K \leq\left|\beta^{\prime}\right|^{L}\left|x^{\prime}+y^{\prime}\right| \leq\left|\beta^{\prime}\right|^{L}\left(\left|x^{\prime}\right|+\left|y^{\prime}\right|\right)<2 H\left|\beta^{\prime}\right|^{L},
$$

which implies (2). Note that the supremum $H$ is never attained, i.e. $\left|z^{\prime}\right|<H$ for all $z \in \mathbb{Z}_{\beta}$. The proof for multiplication is similar.

Remark 3.4. 1. Using the same inequalities as in the proof of Proposition 2.3 we obtain

$$
H \leq[\beta] \frac{1}{1-\left|\beta^{\prime}\right|} .
$$


2. If $\beta^{\prime} \in(0,1)$, then $K=1$. Indeed, for $z=\sum_{i=0}^{n} z_{i} \beta^{i}, z_{0} \neq 0$, one has

$$
z^{\prime}=\sum_{i=0}^{n} z_{i}\left(\beta^{\prime}\right)^{i} \geq z_{0} \geq 1 .
$$

Corollary 3.5. Let $\beta>1$ be an algebraic integer such that at least one of its conjugates, say $\beta^{\prime}$, belongs to $(0,1)$. Then

$$
\left(\frac{1}{\left|\beta^{\prime}\right|}\right)^{L_{\oplus}(\beta)}<\frac{2[\beta]}{1-\beta^{\prime}}, \quad\left(\frac{1}{\left|\beta^{\prime}\right|}\right)^{L_{\odot}(\beta)}<\frac{[\beta]^{2}}{\left(1-\beta^{\prime}\right)^{2}} .
$$

4. Theorem 3.3 for quadratic Pisot numbers. So far we have been interested in $L_{\oplus}(\beta)$ and $L_{\odot}(\beta)$ for general algebraic integers $\beta$. From now on we shall focus on quadratic Pisot numbers. In the quadratic case the Pisot condition implies that $\beta$ is a solution of an equation

$$
\begin{array}{ll}
x^{2}=m x-n, & m, n \in \mathbb{N}, m \geq n+2, \\
x^{2}=m x+n, & m, n \in \mathbb{N}, m \geq n .
\end{array}
$$

We shall try to apply Theorem 3.3 for such $\beta$ and derive the corresponding bounds on $L_{\oplus}(\beta)$ and $L_{\odot}(\beta)$. It will be seen that the situation drastically differs for the two types of quadratic equations.

Note that for $n=1$, the root $\beta$ is a quadratic Pisot unit. For such $\beta$ the values of $L_{\oplus}(\beta)$ and $L_{\odot}(\beta)$ have been determined in [3].

Let us now study the case of $\beta>1$ solving the equation $x^{2}=m x-n$, $m, n \in \mathbb{N}, m \geq n+2$. Note that $[\beta]=m-1$, thus the digits in $\beta$-expansions are $0,1, \ldots, m-1$. The conjugate $\beta^{\prime}$ of $\beta$ satisfies $\beta^{\prime} \in(0,1)$, and the $\beta$ development of unity is $d(1, \beta)=(m-1)(m-n-1)^{\omega}$. For $z \in \mathbb{Z}_{\beta}$, $z=$ $\sum_{i=0}^{n} z_{i} \beta^{i}$ we have

$$
\begin{aligned}
z^{\prime} & =\sum_{i=0}^{n} z_{i}\left(\beta^{\prime}\right)^{i}<(m-1)+(m-2) \beta^{\prime}+(m-2) \beta^{\prime 2}+\ldots \\
& =1+(m-2) \frac{1}{1-\beta^{\prime}}=\frac{\beta(\beta-1)}{\beta-n}=H .
\end{aligned}
$$

Clearly, $\beta(\beta-1) /(\beta-n)$ above is the desired supremum $H$ of Theorem 3.3, since we can construct a sequence of numbers

$$
z_{n}=(m-1) \beta^{0}+\sum_{i=1}^{n}(m-2) \beta^{i} \in \mathbb{Z}_{\beta} \backslash \beta \mathbb{Z}_{\beta}
$$

such that $\lim _{n \rightarrow \infty}\left|z_{n}^{\prime}\right|=H$. For the relation (4) we have considered the admissibility of sequences of digits in $\beta$-expansions. According to Remark 3.4 we have $K=1$, and hence we can use Theorem 3.3 to derive results for $L_{\oplus}(\beta)$ and $L_{\odot}(\beta)$. 
Proposition 4.1. Let $\beta^{2}=m \beta-n, m \geq n+2$. Then

$$
L_{\oplus}(\beta) \leq 3 m \ln m, \quad L_{\odot}(\beta) \leq 4 m \ln m .
$$

In particular, if $n=1$, then $L_{\oplus}(\beta)=L_{\odot}(\beta)=1$.

Proof. Since $K=1$ and $H=\beta(\beta-1) /(\beta-n)=(\beta-1)^{2} /(m-n-1)$ we can estimate

$$
\left(\frac{m-1}{n}\right)^{L_{\oplus}}<\left(\frac{\beta}{n}\right)^{L_{\oplus}}=\left(\frac{1}{\beta^{\prime}}\right)^{L_{\oplus}}<2 \frac{(\beta-1)^{2}}{m-n-1}<2 \frac{(m-1)^{2}}{m-n-1} .
$$

For $n=1$ we obtain directly $L_{\oplus} \leq 1$. For general $n \leq m-2$ we estimate the left hand side of the inequality by

$$
\left(\frac{m-1}{n}\right)^{L_{\oplus}} \geq\left(\frac{m-1}{m-2}\right)^{L_{\oplus}}>e^{L_{\oplus} / m},
$$

where we have used $(1+1 / k)^{k+1}>e$ for $k \in \mathbb{N}$. The right hand side of the inequality is estimated by $m^{3}$. Altogether we get $L_{\oplus}(\beta) \leq 3 m \ln m$. The estimate for $L_{\odot}(\beta)$ is derived analogously, the first step for $n=1$ being

$$
\beta^{L_{\odot}}=\left(\frac{1}{\beta^{\prime}}\right)^{L_{\odot}}<\left(\frac{\beta(\beta-1)}{\beta-1}\right)^{2}=\beta^{2} \Rightarrow L_{\odot} \leq 1 .
$$

In order to show that for $n=1$ we have $L_{\oplus}(\beta)=L_{\odot}(\beta)=1$ it suffices to observe that

$$
((m-1)+(m-1))_{\beta}=(2 \cdot(m-1))_{\beta}=\left(\beta+(m-2)+\frac{1}{\beta}\right)_{\beta}=1(m-2) \bullet 1 .
$$

Let us now study the case of $\beta>1$ solving the equation $x^{2}=m x+n$, $m, n \in \mathbb{N}, m \geq n$. Note that $[\beta]=m$. Therefore the digits in $\beta$-expansions are $0,1, \ldots, m$. The $\beta$-development of unity is $d(1, \beta)=m n$. Now the conjugate $\beta^{\prime}$ of $\beta$ satisfies $\beta^{\prime} \in(-1,0)$. If $w \in \mathbb{Z}_{\beta}, w=\sum_{i=0}^{n} w_{i} \beta^{i}$, we have

$$
\begin{aligned}
\ldots+m{\beta^{\prime}}^{3}+m \beta^{\prime} & <w^{\prime}<m+m{\beta^{\prime}}^{2}+m \beta^{\prime 4}+\ldots, \\
-1 & <w^{\prime}<\frac{m}{1-{\beta^{\prime}}^{2}}=\frac{\beta^{2} m}{m \beta+n-n^{2}}=H .
\end{aligned}
$$

Unfortunately, in this case $K=0$ for all $n \in \mathbb{N}$ except $n=1$. Therefore only for $n=1$ can we use Theorem 3.3 to find $L_{\oplus}(\beta)$ and $L_{\odot}(\beta)$. In this case for $z \in \mathbb{Z}_{\beta}, z=\sum_{i=0}^{n} z_{i} \beta^{i}$ with $z_{0} \neq 0$, we have

$$
\begin{aligned}
z^{\prime} & \geq z_{0}+z_{1} \beta^{\prime}+z_{3} \beta^{3}+z_{5}{\beta^{\prime}}^{5}+\ldots \\
& \geq 1+(m-1) \beta^{\prime}+m{\beta^{\prime}}^{3}+m{\beta^{\prime}}^{5}+\ldots \\
& =1-\beta^{\prime}+\frac{m \beta^{\prime}}{1-\beta^{\prime}}=-\beta^{\prime}=\frac{1}{\beta}=K .
\end{aligned}
$$


Note that $H$ is equal to $\beta$ for $n=1$. Using (2) and (3), we obtain for $m \geq 2$

$$
\left.\begin{array}{l}
\beta^{L_{\oplus}}<2 \beta^{2}<\beta^{3} \\
\beta_{L_{\odot}}<\beta^{3}
\end{array}\right\} \Rightarrow \begin{aligned}
& L_{\oplus}(\beta) \leq 2, \\
& L_{\odot}(\beta) \leq 2 .
\end{aligned}
$$

To prove that $L_{\oplus}(\beta)=L_{\odot}(\beta)=2$ we calculate

$(m+m)_{\beta}=(2 \cdot m)_{\beta}=\left(\beta+(m-1)+\frac{m-1}{\beta}+\frac{1}{\beta^{2}}\right)_{\beta}=1(m-1) \bullet(m-1) 1$.

For $m=1$, i.e. $\beta$ the golden ratio, it is not true that $2 \beta^{2}<\beta^{3}$. A slightly finer discussion is necessary to obtain the exact bound on the number of fractional digits of the sum $x+y$.

In the above considerations we are not able to derive any estimates on $L_{\oplus}(\beta)$ and $L_{\odot}(\beta)$ if $\beta$ is a solution of $x^{2}=m x+n, m, n \in \mathbb{N}, m \geq n \geq 2$. Therefore in the rest of the paper we focus on such quadratic Pisot numbers. First we give an estimate on $L_{\odot}(\beta)$ using $L_{\oplus}(\beta)$ and then we determine the value of $L_{\oplus}(\beta)$.

5. Relation of $L_{\oplus}$ and $L_{\odot}$ for quadratic Pisot numbers. In Section 2 we have shown that $\mathbb{Z}_{\beta}$ can be embedded into a cut-and-project sequence with a suitably chosen window. In our case $\beta$ is a solution of $x^{2}=m x+n, m, n \in \mathbb{N}, m \geq n \geq 2$. Therefore we choose $\Sigma(H)$, where $H=m /\left(1-\beta^{\prime 2}\right)$. We show that a cut-and-project set with arbitrary window can be embedded into a finite union of shifted copies of $\mathbb{Z}_{\beta}$, where the shifts belong to $\mathbb{Z}[\beta]$. In fact, a product $x y$ of $x, y \in \mathbb{Z}_{\beta}$ can be expressed as a sum of a $\beta$-integer and a small rational integer and therefore we can find an upper estimate of $L_{\odot}(\beta)$ using $L_{\oplus}(\beta)$. A similar result can also be proven for non-quadratic Pisot $\beta$. The demonstration is however rather technical.

Theorem 5.1. Let $\beta>1$ be a solution of $x^{2}=m x+n, m, n \in \mathbb{N}$, $m \geq n$, and let $h>0$. Then there exists $p \in \mathbb{N}$ such that

$$
\Sigma(h) \subset \mathbb{Z}_{\beta}+\{-p,-p+1, \ldots,-1,0,1, \ldots, p-1, p\},
$$

where

$$
p \leq h-\beta^{\prime} H=h-\beta^{\prime} \frac{m}{1-\beta^{\prime 2}} .
$$

Proof. Since $\beta$ is a quadratic integer, we can write every power $\beta^{k}$ as an integer combination of 1 and $\beta$. Define $F_{k}, G_{k}$ by

$$
\beta^{k}=F_{k} \beta+G_{k} \text {. }
$$

Since $\beta^{k+1}=\beta\left(F_{k} \beta+G_{k}\right)=F_{k} m \beta+F_{k} n+G_{k} \beta$, the sequences $\left(F_{k}\right)_{k \in \mathbb{N}_{0}}$, $\left(G_{k}\right)_{k \in \mathbb{N}_{0}}$ satisfy $F_{k+1}=m F_{k}+G_{k}, G_{k+1}=n F_{k}$, which gives a recurrence 
relation

$$
F_{k+2}=m F_{k+1}+n F_{k}, \quad \text { where } F_{0}=0, F_{1}=1 .
$$

It is easy to see that every $x \in \mathbb{N}$ can be written in the form $x=\sum_{i=1}^{j} c_{i} F_{i}$, where $c_{i} \in\{0,1, \ldots, m\}$ and $c_{i} c_{i-1}$ is lexicographically smaller than $m n$. The coefficients $c_{j}, c_{j-1}, \ldots, c_{1}$ can be found by the so-called greedy algorithm. Thus $j$ is a number for which $F_{j} \leq x<F_{j+1}$ and $c_{j}:=\left[x F_{j}^{-1}\right]$. We obtain the coefficients $c_{i}, i<j$, by applying the same steps to the integer $\widetilde{x}=x-c_{j} F_{j}$.

Let $z \in \Sigma(h)$, i.e. $z=a+b \beta$ and $\left|z^{\prime}\right|<h$. Since both $\Sigma(h)$ and $\mathbb{Z}_{\beta}$ are symmetric with respect to the origin, it suffices to show the statement for $b \geq 0$. Let $b=\sum_{i=1}^{j} c_{i} F_{i}$. Then

$$
z=\sum_{i=1}^{j} c_{i}\left(F_{i} \beta+G_{i}\right)-\sum_{i=1}^{j} c_{i} G_{i}+a=z_{1}+z_{2},
$$

where $z_{2}:=a-\sum_{i=1}^{j} c_{i} G_{i} \in \mathbb{Z}$ and $z_{1}:=\sum_{i=1}^{j} c_{i} \beta^{i} \in \beta \mathbb{Z}_{\beta} \subset \mathbb{Z}_{\beta}$. Applying the Galois automorphism to the equality $z=z_{1}+z_{2}$ gives $z_{2}=z^{\prime}-z_{1}^{\prime}$. Since $\left|z^{\prime}\right|<h$ and $\left|z_{1}^{\prime}\right|<-\beta^{\prime} H$, the integer $z_{2}$ belongs to the interval $\left(-h+\beta^{\prime} H, h-\beta^{\prime} H\right)$.

\section{Corollary 5.2.}

$$
\mathbb{Z}_{\beta} \mathbb{Z}_{\beta} \subset \mathbb{Z}_{\beta}+\{-p, \ldots, p\}, \text { where } p \leq(m+2)^{4} / 4 .
$$

Proof. Since $\mathbb{Z}_{\beta} \subset \Sigma(H)$, we have $\mathbb{Z}_{\beta} \mathbb{Z}_{\beta} \subset \Sigma\left(H^{2}\right)$. The proof will be completed if we verify that $H^{2}-\beta^{\prime} H \leq \frac{1}{4}(m+2)^{4}$. Let us first show that

$$
\frac{1}{1-\beta^{\prime 2}}<\frac{m+3}{2} \text {. }
$$

We have $-\beta^{\prime}=n / \beta$, thus for $n \leq m-1$,

$$
1-{\beta^{\prime}}^{2}=1-\frac{n^{2}}{\beta^{2}}>1-\frac{n^{2}}{m^{2}} \geq 1-\frac{(m-1)^{2}}{m^{2}}=\frac{2 m-1}{m^{2}} \geq \frac{2}{m+3} .
$$

For $n=m$ the inequality (6) is verified directly using $\beta^{\prime}=\frac{1}{2}\left(m-\sqrt{m^{2}+4 m}\right)$. Therefore

$$
\begin{aligned}
H^{2}-\beta^{\prime} H & \leq H^{2}+H=\frac{m^{2}}{\left(1-\beta^{\prime 2}\right)^{2}}+\frac{m}{1-\beta^{\prime 2}}<\frac{m^{2}(m+3)^{2}}{4}+\frac{m(m+3)}{2} \\
& =\frac{1}{4} m(m+1)(m+2)(m+3) \leq \frac{1}{4}(m+2)^{4} .
\end{aligned}
$$

The above corollary states that a product of two $\beta$-integers can be written as a sum of a $\beta$-integer and a rational integer. Let us derive the number of fractional digits of the $\beta$-expansion of a rational integer $p$.

Lemma 5.3. Let $p \in \mathbb{N}$. Then

$$
\operatorname{fp}_{\beta}(p) \leq\left(1+\log _{2} p\right) L_{\oplus}(\beta) .
$$


Proof. The proof is based on the simple observation that

$$
\operatorname{fp}_{\beta}(x+y) \leq \max \left\{\mathrm{fp}_{\beta}(x), \mathrm{fp}_{\beta}(y)\right\}+L_{\oplus}(\beta),
$$

which in particular gives $\mathrm{fp}_{\beta}(2 x) \leq \mathrm{fp}_{\beta}(x)+L_{\oplus}(\beta)$. Applying the latter $k$ times we obtain $\mathrm{fp}_{\beta}\left(2^{k}\right) \leq k L_{\oplus}(\beta)$. We use induction on $j$ to prove that if $p$ has a binary expansion $p=\sum_{i=0}^{j} a_{i} 2^{i}$ then $\mathrm{fp}_{\beta}(p) \leq(j+1) L_{\oplus}(\beta)$. Using the hypothesis for $p=\sum_{i=0}^{j} a_{i} 2^{i}=2^{j}+\sum_{i=0}^{j-1} a_{i} 2^{i}$ we obtain

$$
\begin{aligned}
\operatorname{fp}_{\beta}(p) & \leq \max \left\{\operatorname{fp}_{\beta}\left(2^{j}\right), \operatorname{fp}_{\beta}\left(\sum_{i=0}^{j-1} a_{i} 2^{i}\right)\right\}+L_{\oplus}(\beta) \\
& \leq \max \left\{j L_{\oplus}(\beta), j L_{\oplus}(\beta)\right\}+L_{\oplus}(\beta)=(j+1) L_{\oplus}(\beta) .
\end{aligned}
$$

The statement of the lemma follows easily from the fact that $j \leq \log _{2} p$.

The following theorem is a simple consequence of Corollary 5.2 and Lemma 5.3.

Theorem 5.4. Let $\beta>1$ be a solution of $x^{2}=m x+n, m, n \in \mathbb{N}$, $m \geq n$. Then

$$
L_{\odot}(\beta) \leq 4 L_{\oplus}(\beta) \log _{2}(m+2) .
$$

Proof. Let $x, y \in \mathbb{Z}_{\beta}$. Using Corollary 5.2, we have $\mathrm{fp}_{\beta}(x y)=\mathrm{fp}_{\beta}(z+p)$ for some $z \in \mathbb{Z}_{\beta}$ and $p \in \mathbb{N}, p \leq \frac{1}{4}(m+2)^{4}$. Now, due to $(7)$,

$$
\begin{aligned}
\operatorname{fp}_{\beta}(z+p) & \leq \operatorname{fp}_{\beta}(p)+L_{\oplus}(\beta) \leq\left(2+\log _{2} p\right) L_{\oplus}(\beta) \\
& \leq\left(2+\log _{2} \frac{(m+2)^{4}}{4}\right) L_{\oplus}(\beta) .
\end{aligned}
$$

The statement of the theorem follows easily.

6. $L_{\oplus}$ for quadratic $\beta$. In this section we obtain an upper bound on $L_{\oplus}(\beta)$. This is done in two steps: first we find an upper bound on $\operatorname{fp}(x+y)$ where $x$ is an arbitrary $\beta$-integer and $y$ is a $\beta$-integer of a specific form. Then we show that any $\beta$-integer can be written as a finite sum of numbers of this specific form. An upper bound on $L_{\oplus}(\beta)$ is obtained by combining both results.

Let $\beta>1$ be a solution of $x^{2}=m x+n, m, n \in \mathbb{N}, m \geq n$. Let $(x)_{\beta}=$ $x_{k} x_{k-1} \ldots x_{1} x_{0} \bullet x_{-1} x_{-2} \ldots x_{-p}$ be a $\beta$-representation of $x$, i.e. $0 \leq x_{i} \leq m$. The $\beta$-representation $(x)_{\beta}$ is a $\beta$-expansion of $x$ if and only if $x_{i} x_{i-1}$ is lexicographically smaller than $m n=d(1, \beta)$ for every $i$.

The following lemma is an easy consequence of the result of Frougny and Solomyak in [4]. It is mentioned here in order to make the article selfcontained.

Lemma 6.1. Let $(x)_{\beta}=x_{k} x_{k-1} \ldots x_{1} x_{0} \bullet x_{-1} x_{-2} \ldots x_{-p}$ be a $\beta$-representation of $x$. Then $\operatorname{fp}_{\beta}(x) \leq p$. 
Proof. If the representation is already in the form of a $\beta$-expansion, then $\mathrm{fp}_{\beta}(x)=p$. Otherwise we can find the largest $j$ such that $x_{j} x_{j-1}$ is lexicographically greater than or equal to $m n$. Since $x_{i} \leq m$ for all $i$, necessarily $x_{j}=m$ and $x_{j-1} \geq n$. Since $j$ was the largest index with this property, $x_{j+1}<m$. Therefore we can define a new representation of $x$ as

$$
(x)_{\beta}=\widetilde{x}_{k} \widetilde{x}_{k-1} \ldots \widetilde{x}_{1} \widetilde{x}_{0} \bullet \widetilde{x}_{-1} \widetilde{x}_{-2} \ldots \widetilde{x}_{-p},
$$

where $\widetilde{x}_{j}:=x_{j}-m, \widetilde{x}_{j-1}:=x_{j-1}-n, \widetilde{x}_{j+1}:=x_{j+1}+1$, and $\widetilde{x}_{i}=x_{i}$ otherwise. In the new representation the sum of digits is strictly smaller than in the previous one. This procedure can be repeated and in finitely many steps we obtain the $\beta$-expansion of $x$. The result follows easily, since in each step the number of digits in the fractional part of the representation does not increase.

Let us first determine a lower bound on $L_{\oplus}(\beta)$. It suffices to find a single example of addition with specified fractional part length. We use the following example.

EXAmple 6.2. Consider $x=m \sum_{i=0}^{k-1} \beta^{2 i}$. Then it can be shown by induction on $k$ that

$$
x+x=\sum_{i=0}^{k-1}\left(A_{k-i} \beta+B_{k-i}\right) \beta^{2 i}+\sum_{i=0}^{k-1}\left(\frac{a_{k-i}}{\beta}+\frac{b_{k-i}}{\beta^{2}}\right) \beta^{-2 i},
$$

where the coefficients $A_{i}, B_{i}, a_{i}$ and $b_{i}, i \in \mathbb{N}$, are defined by

$$
\begin{aligned}
A_{i} & =i(m-n+1)-m+n, \\
B_{i} & =2 m-n-i(m-n+1), \\
a_{i} & =i(m-n+1)-1, \\
b_{i} & =m+1-i(m-n+1) .
\end{aligned}
$$

Formally, we have

$$
x+x=A_{1} B_{1} A_{2} B_{2} \ldots A_{k} B_{k} \bullet a_{k} b_{k} \ldots a_{2} b_{2} a_{1} b_{1} .
$$

The above expression is a $\beta$-expansion if and only if all the coefficients $A_{i}$, $B_{i}, a_{i}$ and $b_{i}$ take values in $\{0,1, \ldots, m\}$ for $i=1, \ldots, k$. This implies the following conditions on $k$ :

$$
\begin{aligned}
k(m-n+1) & \leq m+1, \\
(k-1)(m-n+1) & \leq m-1 .
\end{aligned}
$$

For $m=n$ the latter condition is stronger and the maximal $k$ satisfying it is $k=m$. If on the other hand $m>n$, the first condition is stronger and the maximal $k \in \mathbb{N}$ satisfying it is

$$
k_{0}:=\left[\frac{m+1}{m-n+1}\right] .
$$


COROLlaRY 6.3. Let $\beta$ be the larger solution of $x^{2}=m x+n, m, n \in \mathbb{Z}$, $m \geq n>0$. Then

$$
L_{\oplus}(\beta) \geq \begin{cases}2 m & \text { if } m=n \\ 2 k_{0} & \text { if } m>n\end{cases}
$$

From now on we focus on determining the upper bound for $L_{\oplus}(\beta)$.

LEMMA 6.4. Let $x, y \in \mathbb{Z}_{\beta}, x, y \geq 0$, with $\beta$-expansions

$$
\begin{aligned}
(x)_{\beta} & =x_{l} x_{l-1} \ldots x_{1} x_{0} \bullet, \\
(y)_{\beta} & =y_{k} y_{k-1} \ldots y_{1} y_{0} \bullet,
\end{aligned}
$$

where $y_{i} \leq m-n+1$ for $i=0,1, \ldots, k-2, k-1$. Then the $\beta$-expansion of $x+y$ is

$$
(x+y)_{\beta}=z_{r} z_{r-1} \ldots z_{1} z_{0} \bullet z_{-1} z_{-2},
$$

where

$$
\frac{z_{-1}}{\beta}+\frac{z_{-2}}{\beta^{2}} \in\left\{0, \frac{n}{\beta}, \frac{m-n}{\beta}+\frac{n}{\beta^{2}}\left(=1-\frac{n}{\beta}\right)\right\} .
$$

Proof. We make use of the relation $m+p=\beta+p-1+(m-n) \beta^{-1}+n \beta^{-2}$ for $p \leq m$, i.e. $(m+p)_{\beta}=1(p-1) \bullet(m-n) n$. Symbolically it may be rewritten as

$$
\begin{array}{cc|c}
\multicolumn{1}{c|}{\begin{array}{l} 
\\
+
\end{array}} & \\
\hline 1 & (p-1) & (m-n) n
\end{array}
$$

We proceed by induction on the values of $y$. Let $y=y_{0} \leq m-n+1$. Then, according to (8), the $\beta$-representation of $x+y$ is

$$
(x+y)_{\beta}= \begin{cases}x_{l} \ldots x_{1}\left(x_{0}+y_{0}\right) \bullet & \text { if } x_{0}+y_{0} \leq m \\ x_{l} \ldots\left(x_{1}+1\right)\left(x_{0}+y_{0}-m-1\right) \bullet(m-n) n & \text { if } x_{0}+y_{0}>m .\end{cases}
$$

Note that $x_{1}+1 \leq m$ in the second case, since $x_{1}=m$ implies $x_{0} \leq n-1$, and thus $x_{0}+y_{0} \leq n-1+m-n+1=m$, which is a contradiction.

Now assume that the statement holds for all $\widetilde{y}<y$ satisfying the conditions of the lemma. Suppose that there exists an index $i$ such that $y_{i}>0$ and $x_{i}<m$. Then $x+y=\widetilde{x}+\widetilde{y}$, where according to Lemma $6.1, \widetilde{x}=x+\beta^{i} \in \mathbb{Z}_{\beta}$ and $\widetilde{y}=y-\beta^{i}$ satisfies the conditions of the lemma. We may thus use the induction hypothesis.

Suppose that $y_{i}>0$ implies $x_{i}=m$ for all $i \leq k$. Since $x_{l} x_{l-1} \ldots x_{1} x_{0}$ is an expansion, $x_{i}=m$ implies $x_{i-1} \leq n-1<m$. Thus $y_{i}>0$ implies $y_{i-1}=0$. Since $y_{k}>0$, we have $x_{k}=m$ and $x_{k+1}<m$. Without loss of generality we can consider only the case when $l \leq k+1$. Therefore we have 
the following situation:

$$
\begin{array}{ccccccc}
x_{k+1} & m & x_{k-1} & x_{k-2} & \ldots & x_{1} & x_{0} \\
y_{k} & 0 & y_{k-2} & \ldots & y_{1} & y_{0}
\end{array}
$$

Let $j$ be the smallest integer among $\{1,2, \ldots,[k / 2]\}$ such that $y_{k-2 j}<$ $m-n+1$. Then

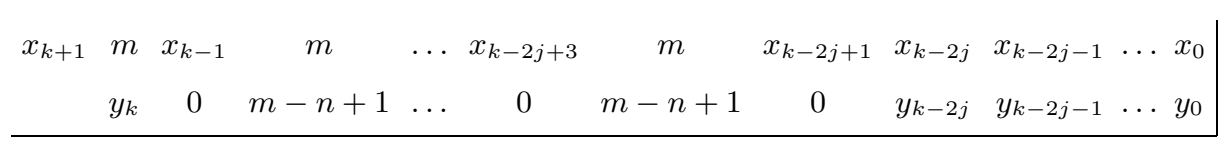

We may check by elementary algebra using the relation $\beta^{2}=m \beta+n$ that

$$
\begin{aligned}
& m \beta^{k}+(m-n+1) \sum_{i=1}^{j-1} \beta^{k-2 i} \\
& =\beta^{k+1}-\beta^{k}+(m-n+1) \beta \sum_{i=1}^{j-1} \beta^{k-2 i}+(m-n) \beta^{k-2 j+1}+n \beta^{k-2 j}
\end{aligned}
$$

Using this relation, we may write the sum $x+y=\widetilde{x}+\widetilde{y}$ as

$$
\begin{array}{rllllllllllll}
\left(x_{k+1}+1\right) & \left(y_{k}-1\right) & \widetilde{x}_{k-1} & m & \ldots & \widetilde{x}_{k-2 j+3} & m & \widetilde{x}_{k-2 j+1} & x_{k-2 j} & x_{k-2 j-1} & \ldots & x_{0} \\
& & & & \left(y_{k-2 j}+n\right) & y_{k-2 j-1} & \ldots & y_{0}
\end{array}
$$

where $\widetilde{x}_{k-2 i+1}=x_{k-2 i+1}+m-n+1$ for $i=1, \ldots, j-1$ and $\widetilde{x}_{k-2 j+1}=$ $x_{k-2 j+1}+m-n$. The first row represents the summand $\widetilde{x}$, the second row the summand $\widetilde{y}$. Due to (9) we have $x+y=\widetilde{x}+\widetilde{y}$. Obviously $\widetilde{x}, \widetilde{y} \in \mathbb{Z}_{\beta}$, the digits of $\widetilde{y}$ are $\leq m-n+1$, except its first non-zero digit from the left. We have $\widetilde{y}<y$ and thus we may use the induction hypothesis.

It remains to solve the case where $y_{k-2 i}=m-n+1$ for all $i \in$ $\{1,2, \ldots,[k / 2]\}$. Then either

$$
y=y_{k} 0(m-n+1) 0(m-n+1) \ldots 0(m-n+1),
$$

or

$$
y=y_{k} 0(m-n+1) 0(m-n+1) \ldots 0(m-n+1) 0
$$

i.e.

$$
y=y_{k} \beta^{k}+(m-n+1) \sum_{i=1}^{[k / 2]} \beta^{k-2 i}
$$

for $k$ even or odd. We may deduce from (9) that the results of the addition $x+y$ have fractional parts $1-n / \beta$ and $n / \beta$ respectively. This completes the proof. 
Lemma 6.5. Let $x, y \in \mathbb{Z}_{\beta}, x>y \geq 0$. Then

$$
x-y=\left\{\begin{array}{l}
z \\
z+(n-1) \sum_{i=0}^{k} \beta^{2 i}+1 \\
z+(n-1) \sum_{i=1}^{k} \beta^{2 i-1}+\frac{n}{\beta}
\end{array} \quad \text { with } z \in \mathbb{Z}_{\beta}, z \geq 0, k \geq 0 .\right.
$$

Proof. First note that for every $x \in \mathbb{Z}_{\beta}$ there exists a $\beta$-representation $(x)_{\beta}=x_{l} \ldots x_{1} x_{0} \bullet$ such that $x_{i}+x_{i-1}>0$ for all $0<i \leq l$, i.e. the $\beta$-representation is "dense". The dense form can be found by the following procedure: Find the first pair of zeros from the left, say $x_{i}=x_{i-1}=0$, $x_{i+1}>0$. Put $\widetilde{x}_{i+1}=x_{i+1}-1, \widetilde{x}_{i}=m, \widetilde{x}_{i-1}=n$, and $\widetilde{x}_{j}=x_{j}$ for all other $0 \leq j \leq l$. The new $\beta$-representation $(x)_{\beta}=\widetilde{x}_{l} \ldots \widetilde{x}_{1} \widetilde{x}_{0} \bullet$ has strictly lower number of vanishing coefficients. Thus the procedure is finite.

The proof of the lemma is by induction on the value of $y$. Without loss of generality we may assume that both $(x)_{\beta}=x_{l} \ldots x_{1} x_{0} \bullet$ and $(y)_{\beta}=$ $y_{k} \ldots y_{1} y_{0} \bullet$ are written in their dense form.

Assume that there is an index $i$ such that both $x_{i}$ and $y_{i}$ are non-zero. Then $x-y=\widetilde{x}-\widetilde{y}$, where $\widetilde{x}=x-\beta^{i}$ and $\widetilde{y}=y-\beta^{i}$. Clearly, $\widetilde{x}, \widetilde{y} \in \mathbb{Z}_{\beta}$ and $\widetilde{y}<y$, thus we may use the induction hypothesis.

Assume that $y_{i}>0$ implies $x_{i}=0$ for all indices $i$. Since $x_{i}+x_{i-1}>0$, we have $y_{i-1}=0$. Since $y_{k}>0$, we have $x_{k}=0$ and $x_{k+1}>0$. Without loss of generality we consider $l=k+1$ and $x_{k+1}=1$. Since both $x$ and $y$ are in their dense form, the remaining cases are as follows. First assume that the maximal index $k$ such that $y_{k}$ is non-zero, is even. We have $x-y$ equal to

\begin{tabular}{cccccccc|c}
1 & 0 & $x_{k-1}$ & 0 & $x_{k-3}$ & $\ldots$ & $x_{1}$ & 0 & \\
- & $y_{k}$ & 0 & $y_{k-2}$ & 0 & $\ldots$ & 0 & $y_{0}$ & \\
\hline 1 & 0 & $x_{k-1}$ & 0 & $x_{k-3}$ & $\ldots$ & $x_{1}$ & 0 & \\
-1 & 0 & 0 & 0 & 0 & $\ldots$ & 0 & 0 & \\
+ & $m$ & $(n-1)$ & $m$ & $(n-1)$ & $\ldots$ & $(n-1)$ & $m$ & $n$ \\
- & $y_{k}$ & 0 & $y_{k-2}$ & 0 & $\ldots$ & 0 & $y_{0}$ & \\
\hline & $\left(m-y_{k}\right)$ & $x_{k-1}$ & $\left(m-y_{k-2}\right)$ & $x_{k-3}$ & $\ldots$ & $x_{1}$ & $\left(m-y_{0}\right)$ & \\
+ & & $(n-1)$ & 0 & $(n-1)$ & $\ldots$ & $(n-1)$ & 0 & $n$ \\
\hline \hline
\end{tabular}

which corresponds to the statement of the lemma. For $k$ odd we may write 
similarly that $x-y$ equals

\begin{tabular}{|c|c|c|c|c|c|c|c|c|}
\hline 1 & 0 & $x_{k-1}$ & 0 & $x_{k-3}$ & $\ldots$ & $x_{2}$ & 0 & $x_{0}$ \\
\hline- & $y_{k}$ & 0 & $y_{k-2}$ & 0 & $\cdots$ & 0 & $y_{1}$ & 0 \\
\hline & $\left(m-y_{k}\right)$ & $x_{k-1}$ & $\left(m-y_{k-2}\right)$ & $x_{k-3}$ & & $x_{2}$ & $\left(m-y_{1}\right)$ & $x_{0}$ \\
\hline+ & & $(n-1)$ & 0 & $(n-1)$ & $\ldots$ & $(n-1)$ & 0 & $n$ \\
\hline
\end{tabular}

which is of the desired form.

THEOREM 6.6. Let $\beta$ be the larger solution of $x^{2}=m x+n, m, n \in \mathbb{Z}$, $m \geq n>0$. Then

$$
L_{\oplus}(\beta)=2 m \quad \text { if } m=n
$$

and

$$
2\left\lfloor\frac{m+1}{m-n+1}\right\rfloor \leq L_{\oplus}(\beta) \leq 2\left\lceil\frac{m}{m-n+1}\right\rceil \text { if } m>n .
$$

Proof. Let $x, y \in \mathbb{Z}_{\beta}, x y>0$. Every $y$ splits as $y=y_{(1)}+\ldots+y_{(s)}$, for some $s$, where the summands $y_{(i)}$ have digits $\leq m-n+1$, and thus satisfy the assumptions of Lemma 6.4 . We can always choose $y_{(i)}$ in such a way that the sum has at most

$$
s_{0}:=\left\lceil\frac{m}{m-n+1}\right\rceil
$$

non-vanishing summands. Lemma 6.4 then implies that

$$
\mathrm{fp}_{\beta}(x+y) \leq 2 s_{0}
$$

Now let $x y<0$, without loss of generality $x>-y$. Then, according to Lemma $6.5, x+y$ can be written either as $z+w$ for some $0 \leq z, w \in \mathbb{Z}_{\beta}$, or

$$
x+y=z+(n-1) \sum_{i=1}^{k} \beta^{2 i-1}+n / \beta \quad \text { for } 0 \leq z \in \mathbb{Z}_{\beta} .
$$

The sum $(n-1) \sum_{i=1}^{k} \beta^{2 i-1}$ can be written as addition of $\left\lceil\frac{n-1}{m-n+1}\right\rceil=s_{0}-1$ summands with digits $\leq m-n+1$. Therefore

$$
\operatorname{fp}_{\beta}\left(z+(n-1) \sum_{i=1}^{k} \beta^{2 i-1}\right) \leq 2\left(s_{0}-1\right)
$$

Adding $n / \beta$ to the result may yield only two more fractional digits (cf. Lemma 6.4).

Thus the proof for the upper bound on $L_{\oplus}(\beta)$ is finished. The lower bound of $L_{\oplus}(\beta)$ is given by Corollary 6.3 . 
The last two sections were devoted to the study of arithmetic of $\beta$ expansions for $\beta>1$ a solution of $x^{2}=m x+n, m, n \in \mathbb{N}, m \geq n$. This is the case where Theorem 3.3 does not provide us with any results, since $K=0$. Let us comment on the results obtained in Sections 5 and 6 :

1. The lower and upper bounds for $L_{\oplus}(\beta)$ found in Theorem 6.6 differ at most by 2 . They coincide if and only if

$$
m-n+1 \text { divides } m \text { or } m+1 \text {. }
$$

Based on observation, we conjecture that for $m>n$ we actually have $L_{\oplus}(\beta)=2 k_{0}$. We also note that for $m>n$ the results of subtraction $x-y$, where $x, y>0$, have lower numbers of fractional digits than addition, more precisely, $\mathrm{fp}_{\beta}(x-y) \leq 2 k_{0}-1$.

2. According to Theorem 5.4 we may use the bound on $L_{\oplus}(\beta)$ to derive an upper estimate on $L_{\odot}(\beta)$. For example for $m=n$ this gives

$$
L_{\odot}(\beta) \leq 8 m\left(\log _{2}(m+2)\right) .
$$

Acknowledgements. L.S.G. is grateful for the hospitality of the Department of Mathematics, Faculty of Nuclear Sciences and Physical Engineering of the Czech Technical University, where the work was done. Z.M. and E.P. are grateful for the hospitality of the Centre de recherches mathématiques, Université de Montréal. The work was partially supported by the Grant Agency of the Czech Republic GA 201/01/0130; L.S.G. and Z.M. acknowledge the financial support of NSERC of Canada.

\section{References}

[1] S. Akiyama, Self affine tiling and Pisot numeration system, in: Number Theory and its Applications, K. Győry and S. Kanemitsu (eds.), Kluwer, 1999, 7-17.

[2] D. W. Boyd, On the beta expansions for Salem numbers of degree 6, Math. Comp. 65 (1996), 861-875; Salem numbers of degree four have periodic expansions, in: Théorie des nombres (Québec, PQ, 1987), de Gruyter, Berlin, 1989, 57-64.

[3] Č. Burdík, Ch. Frougny, J. P. Gazeau and R. Krejcar, Beta-integers as natural counting systems for quasicrystals, J. Phys. A 31 (1998), 6449-6472.

[4] Ch. Frougny and B. Solomyak, Finite $\beta$-expansions, Ergodic Theory Dynam. Systems 12 (1994), 713-723.

[5] R. V. Moody, Meyer sets and their duals, in: Mathematics of Long Range Aperiodic Order (Waterloo, 1996), R. V. Moody (ed.), Kluwer, 1996, 403-441.

[6] W. Parry, On the $\beta$-expansions of real numbers, Acta Math. Acad. Sci. Hungar. 11 (1960), 401-416.

[7] A. Rényi, Representations for real numbers and their ergodic properties, ibid. 8 (1957), 477-493.

[8] K. Schmidt, On periodic expansions of Pisot numbers and Salem numbers, Bull. London Math. Soc. 12 (1980), 269-278. 
[9] B. Solomyak, Conjugates of beta-numbers and the zero-free domain for a class of analytic functions, Proc. London Math. Soc. 68 (1994), 477-498.

[10] J.-L. Verger-Gaugry and J. P. Gazeau, Geometric study of the set of beta-integers for a Perron number and mathematical quasicrystals, J. Théor. Nombres Bordeaux, to appear.

Department of Mathematics and Statistics

McGill University

Burnside Hall, 805 Sherbrooke Street West

Montréal (Québec) H3A 2K6, Canada

E-mail: guimond@math.mcgill.ca
Department of Mathematics Faculty of Nuclear Sciences and Physical Engineering Czech Technical University Trojanova 13 12000 Praha 2, Czech Republic E-mail: masakova@km1.fjif.cvut.cz pelantova@km1.fjfi.cvut.cz

Received on 11.2.2002

and in revised form on 9.4.2003 